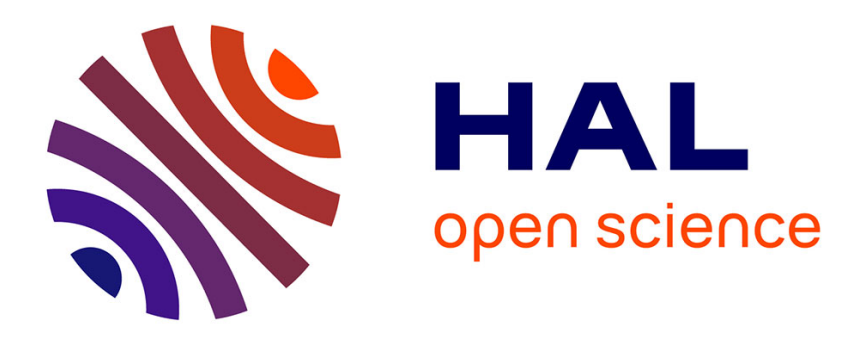

\title{
THE ABSOLUTE PARAMETRIC INSTABILITIES AND THE PLASMA HEATING
}

\author{
V. Silin
}

\section{To cite this version:}

V. Silin. THE ABSOLUTE PARAMETRIC INSTABILITIES AND THE PLASMA HEATING. Journal de Physique Colloques, 1977, 38 (C6), pp.C6-153-C6-159. 10.1051/jphyscol:1977614 . jpa00217199

\section{HAL Id: jpa-00217199 \\ https://hal.science/jpa-00217199}

Submitted on 1 Jan 1977

HAL is a multi-disciplinary open access archive for the deposit and dissemination of scientific research documents, whether they are published or not. The documents may come from teaching and research institutions in France or abroad, or from public or private research centers.
L'archive ouverte pluridisciplinaire HAL, est destinée au dépôt et à la diffusion de documents scientifiques de niveau recherche, publiés ou non, émanant des établissements d'enseignement et de recherche français ou étrangers, des laboratoires publics ou privés. 


\title{
THE ABSOLUTE PARAMETRIC INSTABILITIES AND THE PLASMA HEATING
}

\author{
V. P. SILIN \\ P. N. Lebedev's Physical Institute, Academy of Sciences USSR, Moscow, U. S. S. R.
}

\begin{abstract}
Résumé. - Nous discutons des conditions des instabilités paramétriques dans le plasma inhomogène, leurs seuils d'apparition et la localisation spatiale des ondes croissantes. En outre, en se basant sur la théorie de turbulence faible qui tient compte de l'influence de la largeur spectrale finie sur le seuil de l'instabilité paramétrique secondaire, nous déterminons le niveau de la turbulence du plasma et les fréquences de collision effectives qui rendent compte du taux d'absorption anormal du champ de pompe.
\end{abstract}

\begin{abstract}
We discuss the conditions of the absolute parametric instabilities, their thresholds and the space region of the growing waves localization in the inhomogeneous plasma. Basing on the theory of the weak turbulence that takes into account the finite spectral width influence on the threshold of the secondary parametric instability we determine the level of plasma turbulence and the effective collision frequencies which describe the anomalous absorption rate of the pumping field.
\end{abstract}

Introduction. - Parametric plasma instabilities are of particular interest to us in connection with the possibilities of the increase in the electromagnetic field energy absorption rate. From this standpoint we shall discuss some theoretical results obtained recently by the Department of Plasma Phenomena Theory of the Lebedev Physical Institute, Academy of Sciences of the USSR.

The first set of questions deals with the linear theory of parametric instabilities in space inhomogeneous plasma. Here we consider the task of revealing the conditions for the absolute instabilities, their thresholds and, what we think very important, the plasma disturbance space location arising as the result of such instabilities.

The second set of questions is connected with the nonlinear theory of the parametric turbulence plasma state. Here we are particularly interested in understanding the near real conditions under which we can depend on the strong electromagnetic radiation absorption predictions in terms of the weak turbulence theory. A theoretical conclusion about such a possibility requires answers both in the linear theory of an inhomogeneous plasma and in the nonlinear theory of the turbulent state.

1. Some questions of space inhomogeneous plasma linear theory. - In this part we shall discuss some theoretical results of such instabilities as the transverse electromagnetic wave transformation in two electron plasma waves ( $t \rightarrow 2 l$, two plasmon decay), the conversion into one electron Langmuir wave $(t \rightarrow l+a$, aperiodic instability), the transformation in the electron Langmuir wave and ion sound wave ( $t \rightarrow l+s$, parametric decay), and also the parametric electromagnetic field trap instability, which may be considered separately.

The most significant questions of our treatment are, first of all, the etablishment of the fact of absolute instability, which is connected with the possibility of existence of spatially localized perturbations, and, secondly, the determination of the region of instability occurrence.

In our discussion of parametric instabilities in the spatial nonuniform plasma, the electron (and also ion) density is considered to be a linear function of the $X$-coordinate :

$$
n=n_{0}\left(\text { const }+\frac{X}{L}\right)
$$

where $L$ is the characteristic nonuniformity range. The spatial dependence of the temperature is neglected.

A powerful tool of a plasma waves theoretical study in the nonhomogeneous case is a geometrical optics approximation which permits us to use the following form of the plasma perturbations spatial dependence :

$$
\exp \left\{i K_{y} y+i \mathrm{~K}_{z} Z+i \int \mathrm{d} \mathbf{X} K_{x}(X)\right\} .
$$

Then it appears that we can use the parametric instability dispersion equation of homogeneous plasma 
theory as an eiconal equation with the only exception being the wave vector $\mathbf{k}$ having the components $\overline{\overline{\mathbf{\kappa}}}_{x}(X), \overline{\overline{\boldsymbol{\kappa}}}_{y}, \overline{\mathbf{\kappa}}_{z}$ and the plasma density being a coordinate function.

The eiconal equation of parametric instability arising near a quarter of the critical plasma density $\left(\omega_{0} \sim 2 \omega_{\mathrm{Lc}}\right)$ and corresponding to two plasmon decay of pumping wave has the form [1] :

$$
\left[1+\delta \varepsilon_{\mathrm{e}}(\omega, \overline{\overline{\mathbf{k}}})\right]\left[1+\delta \varepsilon_{\mathrm{e}}\left(\omega-\omega_{0}, \overline{\overline{\mathbf{k}}}\right)\right]=\frac{\overline{\mathbf{\kappa}}_{x}^{2} \overline{\mathbf{\kappa}}_{y}^{2}}{\overline{\overline{\mathbf{\kappa}}}^{4}}|K|^{2},
$$

where

$$
\begin{aligned}
K & =\frac{i}{2} \frac{\mathrm{d}}{\mathrm{d} X}\left[r_{\mathrm{E}}(X) \exp \left(i \int \kappa_{0} \mathrm{~d} X\right)\right], \\
r_{\mathrm{E}} & \equiv \frac{\overline{\overline{\mathbf{v}}}_{\mathrm{E}}}{\omega_{0}}=\frac{e E_{0}(X)}{m_{\mathrm{e}} \omega_{0}^{2}}, \\
\delta \varepsilon_{\mathrm{e}}(\omega, \overline{\overline{\mathbf{\kappa}}}) & =-\frac{\omega_{\mathrm{Le}}^{2}(X)}{\omega^{2}}\left[1+\frac{2 \tilde{i}}{\omega}+3 \overline{\bar{\kappa}}^{2} r_{\mathrm{De}}^{2}\right], \\
r_{\mathrm{Dc}}^{2} & =\frac{\mathrm{X} T_{\mathrm{e}}}{4 \pi e^{2} n_{\mathrm{e}}} .
\end{aligned}
$$

The pump field is polarized along the $y$-axis

$$
E_{y}(X, t)=E_{0}(X) \sin \left(\omega_{0} t-\int \overline{\boldsymbol{\kappa}}_{0}(X) \mathrm{d} X\right) .
$$

The investigations carried out in $[2,3,4]$ have demonstrated that two plasma waves - the result of two plasmon decay - are localized in a small space region with linear dimension

$$
\sim \frac{v_{\mathrm{E}}}{c} L .
$$

The whole length of the region of plasmon localization with every $\kappa_{\perp}$ can be much larger and is defined by the permitted mismatch of resonance using the following formula :

$$
\Delta \overline{\overline{\mathbf{X}}} \sim 3 L r_{\mathrm{De}}^{2}\left(\kappa_{\max }^{2}-\kappa_{\min }^{2}\right) .
$$

When the pump field is several times the threshold value we obtain $\kappa_{\max } \gg \kappa_{\min }$. Therefore it is sufficient to know $\kappa_{\max }$ in order to determine the length (1.5). At the same time, it should be noted that in the case of short-wave length plasmons the space inhomogeneity is not important. That is why for short waves the marginal field of instability is determined as a result of homogeneous plasma theory [5] (compare also $[4,6])$ :

$$
\frac{v_{\mathrm{E}}}{c}=\frac{8}{\sqrt{3}} \frac{\tilde{\gamma}(\kappa)}{\omega_{0}}
$$

where $\tilde{\gamma}$ is the Langmuir plasma wave decrement of Landau damping exponentially depending on the wave vector. According to this [4],

$$
\Delta X \sim L\left\{\ln \frac{c}{v_{\mathrm{E}}}\right\}^{-1} .
$$

Therefore, the whole region of two plasmon instability location can occupy a significant part of the nonuniform plasma profile (because of $v_{\mathrm{E}} / c \gg v_{\mathrm{ei}} / \omega_{0}$ ).

One should not imagine that plasma space inhomogeneity does not play any role. First, the fact of space localized plasmon existence is directly connected with plasma inhomogeneity. Secondly, space inhomogeneity can significantly increase $[4,7]$ the instability threshold from $v_{\mathrm{E}} / c \sim v_{\mathrm{ei}} / \omega_{0}$ up to

$$
v_{\mathrm{E}} / c \sim v_{\mathrm{Te}} / \sqrt{L \omega c} .
$$

According to paper [2], the marginal intensity of the pump wave corresponding to the onset of instability increases with the wave length of plasmons. Therefore, according to [4], if the absolute parametric instability is mainly responsible for $3 / 2 \omega_{0}$ harmonic generation and if there are reasons which restrict spectral redistribution of turbulence into the region of long enough waves (which can be connected with space inhomogeneity also), then two thresholds of $3 / 2 \omega_{0}$ harmonic generation may be experimentally observed.

The eiconal equation of parametric instability for the space region near critical density, where $\omega_{0} \simeq \omega_{\text {Le }}$, can be written in the form [1]

$$
\begin{aligned}
\frac{1}{\delta \varepsilon_{\mathrm{i}}(\omega, \kappa)} & +\frac{1}{1+\delta \varepsilon_{\mathrm{e}}(\omega, \kappa)}+\frac{\left(\mathbf{k r}_{\mathrm{E}}\right)^{2}}{4} \\
& \times\left[\frac{1}{\varepsilon\left(\omega+\omega_{0}, \kappa\right)}+\frac{1}{\varepsilon\left(\omega-\omega_{0}, \kappa\right)}\right]=0 .
\end{aligned}
$$

The theory is very simple in the case of aperiodic instability $(t \rightarrow l+a)$ when for the marginal condition the eiconal equation is [1] :

$\frac{\kappa_{y}^{2} r_{\mathrm{E}}^{2}(X)}{\left[\kappa_{x}^{2}(X)+\kappa_{\perp}^{2}\right]\left(r_{\mathrm{De}}^{2}+r_{\mathrm{Di}}^{2}\right)}=-4 \frac{\tilde{\gamma}^{2}+\left(\Delta \omega_{0}\right)^{2}}{\omega_{0} \Delta \omega_{0}}$

where $\kappa_{\perp}^{2}=\kappa_{y}^{2}+\kappa_{z}^{2}$ and

$$
\Delta \omega_{0}=-\frac{1}{2} \omega_{0}\left\{\frac{X}{L}+3\left[\kappa_{x}^{2}(X)+\kappa_{\perp}^{2}\right] r_{\mathrm{De}}^{2}\right\} .
$$

Let us write equation (1.9) in the following form :

$$
\begin{aligned}
\frac{X}{L}= & -3\left(\kappa_{x}^{2}+\kappa_{\perp}^{2}\right) r_{\mathrm{De}}^{2}+\frac{\kappa_{y}^{2} r_{\mathrm{E}}^{2}}{4\left(\kappa_{x}^{2}+\kappa_{\perp}^{2}\right)\left(r_{\mathrm{De}}^{2}+r_{\mathrm{Di}}^{2}\right)} \\
& \pm \sqrt{\left[\frac{\kappa_{y}^{2} r_{\mathrm{E}}^{2}}{4\left(\kappa_{x}^{2}+\kappa_{\perp}^{2}\right)\left(r_{\mathrm{De}}^{2}+r_{D i}^{2}\right)}\right]^{2}-\frac{4 \tilde{\gamma}^{2}}{\omega_{0}^{2}}}
\end{aligned}
$$


From the last equation it follows that there are two purely real turning points

$$
\kappa_{x}^{2}\left(X_{t}\right)=0 .
$$

Thus, we can talk about the existence of plasma oscillation localized between these points and therefore about absolute instability.

On the other hand, equation (1.9) can be also used directly for writing the dispersion equation :

$$
(2 n+1) \pi=-\int_{-\kappa_{x}(b)}^{+\kappa_{x}(b)} \mathrm{d} \kappa_{x}\left(X_{+}-X_{-}\right)
$$

where the integration is between the transformation points determined by the formula :

$$
\left[\kappa_{x}^{2}(b)\right]=-\kappa_{\perp}^{2}+\frac{\kappa_{y}^{2} r_{\mathrm{E}}^{2}}{8\left(r_{\mathbf{D c}}^{2}+r_{\mathbf{D i}}^{2}\right)} \frac{\omega_{0}}{\tilde{\gamma}} .
$$

From equation (1.11), we have in the limits of weak or strong inhomogeneity $[8,9]$

$$
\frac{\kappa_{y}^{2} r_{\mathrm{E}}^{2}}{\kappa_{\perp}^{2}\left(r_{\mathrm{De}}^{2}+r_{\mathrm{Di}}^{2}\right)}=\frac{8 \tilde{\gamma}}{\omega_{0}} \text { or } \frac{2(2 n+1)}{\kappa_{\perp} L} \text {. }
$$

The threshold pump field of aperiodic instability is determined by the larger value of these two right members.

The length of the localization region of plasmon with fixed $\kappa_{\perp}$ in the case of strong inhomogeneity can be obtained from equation (1.10)

$$
\Delta \overline{\mathbf{X}}_{\mathrm{t}}=\frac{2 n+1}{\kappa_{\perp}} .
$$

Therefore, it is possible to neglect the space dependence of the pump wave always when the wavelengths of the plasmons are small in comparison with the pump wavelength in plasma $\left(\kappa_{\perp} \hbar_{0} \gg\left(\hbar_{0} / L\right)^{1 / 3}\right)$. On the other hand the whole length of the aperiodic instability region can be significantly larger. Accordingly to (1.10), such size is defined by short-wavelength plasmons for which the spatial inhomogeneity is not important. As a result, we have

$$
\Delta \overline{\mathbf{X}} \sim L\left\{\ln \frac{r_{\mathrm{De}}^{2}+r_{\mathrm{Di}}^{2}}{r_{\mathrm{E}}^{2}}\right\}^{-1} .
$$

This length may constitute a significant part of the plasma profile.

The absolute instability connected with parametric decay $t \rightarrow l+s$ is also described by eiconal equation (1.8). Immediately, we should like to point out that such instability is an absolute one due to the space dependence of pump field [10]. The growing plasma waves are localized in a spatially small region of small length compared with the scale of the pump field nonuniformity in the plasma $\sim \lambda_{0}\left(L / \lambda_{0}\right)^{1 / 3} \xi_{\mathrm{m}}^{-1 / 2}$. For the standing pump wave, $\xi_{\mathrm{m}}$ are the coordinates of Airy function maxima.
Having determined the transverse components of plasmon wave vector from the relation :

$$
\kappa_{\perp}^{2} r_{\mathrm{De}}^{2}+\frac{2 \omega}{\omega_{0}}-\frac{\xi_{\mathrm{m}}}{3}\left(\frac{\lambda_{0}}{L}\right)^{2 / 3}=0
$$

one may take into account only the last term in the square brackets of equation (1.8). As a result, for the marginal condition of parametric decay instability, the eiconal equation takes the form :

$$
\begin{aligned}
\kappa_{x}^{4} & +2 \kappa_{x}^{2}\left[\frac{i \tilde{\gamma}}{3 \omega_{0} r_{\mathrm{De}}^{2}}-\frac{i \gamma_{\mathrm{s}}+\delta \omega}{\omega_{\mathrm{Li}} r_{\mathrm{De}}} \kappa_{\perp}\right]+ \\
& +\frac{4 \tilde{\gamma}\left(\gamma_{\mathrm{s}}-i \delta \omega\right) \kappa_{\perp}}{3 \omega_{0} \omega_{\mathrm{Li}} r_{\mathrm{De}}^{3}}-\frac{v_{\mathrm{E}}^{2}(X) \kappa_{\perp}^{2} \sin ^{2} \varphi}{12 v_{\mathrm{Te}}^{2} r_{\mathrm{De}}^{2}}=0
\end{aligned}
$$

where

$$
\kappa_{y}^{2}=\kappa_{\perp}^{2} \sin ^{2} \varphi, \quad \omega^{2}-\kappa_{\perp}^{2} v_{s}^{2}=2 \kappa_{\perp} v_{s} \delta \omega,
$$

$\tilde{\gamma}$ is the decrement of the electron Langmuir waves and $\gamma_{s}$ is the decrement of ion sound waves.

In view of the small length of the plasmon space localization region near the maxima of $v_{\mathrm{E}}^{2}(X)$, we shall use the approximation

$$
v_{\mathrm{E}}^{2}(X)=v_{\mathrm{E}}^{2}(m)\left\{1-C_{\mathrm{m}}\left(\xi-\xi_{\mathrm{m}}\right)^{2}\right\} .
$$

Here $C_{\mathrm{m}}=\xi_{\mathrm{m}}$ for standing pump wave (Airy function) and can be much smaller when the absorption is significant enough. The turning points $\left(\kappa_{x}^{2}=0\right)$ are determined by equation $\left({ }^{1}\right)$

$C_{\mathrm{m}}\left(\xi_{\mathrm{t}}-\xi_{\mathrm{m}}\right)^{2}=1-\frac{4 \tilde{\gamma} \omega_{\mathrm{Li}} v_{\mathrm{Te}}^{2} \sqrt{2 \pi}}{v_{\mathrm{E}}^{2}(m) \omega_{0}^{2} \sin ^{2} \varphi}+\frac{i \delta \omega}{\gamma_{\mathrm{s}}}$.

Under the condition of smallness of the left member of formula (1.18), it follows, first, that the frequency correction $\delta \omega$ is smaller than the ion sound decrement $\left(\delta \omega \ll \gamma_{\mathrm{s}}\right)$ and, secondly, that $v_{\mathrm{E}}^{2}(m)$ is near the marginal value corresponding to the homogeneous plasma theory result :

$\sin ^{2} \varphi \frac{v_{\mathrm{E}}^{2}(m)}{v_{\mathrm{Te}}^{2}}=\frac{4 \sqrt{2 \pi} \tilde{\gamma} \omega_{\mathrm{Li}}}{\omega_{0}^{2}}(1+\delta), \quad|\delta| \ll 1$.

It should be noted that the possibility of the parametric decay $t \rightarrow l+s$ is connected with the possibility of neglecting the second term in the square brackets in eiconal equation (1.8). This requires fulfillment of the condition for smallness of the localization region of this instability determined by the left member of inequality

$$
\left(\frac{\lambda_{0}}{L}\right)^{2 / 3}\left|\xi_{t}-\xi_{\mathrm{m}}\right| \ll \frac{\tilde{\gamma}}{\omega_{0}} \ll \frac{\omega_{\mathrm{Li}}}{\omega_{0}} \xi_{\mathrm{m}}\left(\frac{\lambda_{0}}{L}\right)^{2 / 3} .
$$

(1) About Stokes lines see Appendix. 
The right member of this inequality is connected only with the restriction of this text that permits us to write the following simple equation

$$
\kappa_{x}^{2}=i \frac{2 \tilde{\gamma} C_{\mathrm{m}}}{3 \omega_{0} r_{\mathrm{De}}^{2}}\left[\left(\xi_{\mathrm{t}}-\xi_{\mathrm{m}}\right)^{2}-\left(\xi-\xi_{\mathrm{m}}\right)^{2}\right] .
$$

Then, by means of dispersion equation

$$
\pi\left(n+\frac{1}{2}\right)=\left(\hat{\lambda}_{0}^{2} L\right)^{1 / 3} \int_{-\left(\xi_{t}-\xi_{m}\right)}^{+\left(\xi_{t}-\xi_{m}\right)} \kappa_{x} \mathrm{~d}\left(\xi-\xi_{m}\right)
$$

we can obtain an answer to the question of absolute instability.

Thus, we have $\delta \omega=-\gamma_{\mathrm{s}} \delta$ and

$$
\delta=\left(n+\frac{1}{2}\right) \sqrt{C_{\mathrm{m}}} \frac{v_{\mathrm{Te}}}{c}\left(\frac{\lambda_{0}}{L}\right)^{1 / 3}\left(\frac{3 \omega_{0}}{\tilde{\gamma}}\right)^{1 / 2} .
$$

We have also for the turning points :

$$
C_{\mathrm{m}}\left(\xi_{\mathrm{t}}-\xi_{\mathrm{m}}\right)^{2}=(1-i) \delta .
$$

The condition $\delta \ll 1$ gives us the restriction on the right member of formula (1.21) and on the value of $C_{\mathrm{m}}$. Moreover condition (1.20) takes the following form :

$$
\begin{aligned}
\frac{1}{\xi_{\mathrm{m}}^{1 / 5}}\left(n+\frac{1}{2}\right)^{2 / 5}\left(\frac{3 v_{\mathrm{Te}}^{2}}{16 c^{2}}\right)^{1 / 5}\left(\frac{\lambda_{0}}{L}\right)^{2 / 3} & < \\
<\frac{\tilde{y}}{\omega_{0}} & <\frac{\omega_{\mathrm{Li}}}{\omega_{0}} \xi_{\mathrm{m}}\left(\frac{\lambda_{0}}{L}\right)^{2 / 3} .
\end{aligned}
$$

These conditions are compatible only when $\xi_{\mathrm{m}}$ satisfies the inequality

$$
\begin{aligned}
\left(n+\frac{1}{2}\right) & \left.\frac{v_{\mathrm{Te}}}{c}\left(\frac{\lambda_{0}}{L}\right)^{1 / 3} \sqrt{\frac{3 \omega_{0}}{\tilde{\gamma}}}\right\}^{-2} \gg \xi_{m} \gg \\
& \gg\left(\frac{\omega_{0}}{\omega_{\mathrm{Li}}}\right)^{5 / 6}\left(n+\frac{1}{2}\right)^{1 / 3}\left(\frac{3 v_{\mathrm{Te}}^{2}}{16 c^{2}}\right)^{1 / 6} \\
\frac{\tilde{\gamma}}{\omega_{0}} & \gg\left(\frac{\lambda_{0}}{L}\right)^{2 / 3}\left(\frac{3 v_{\mathrm{Te}}^{2}}{c^{2}}\right)^{7 / 6}\left(\frac{3 \omega_{0}}{\omega_{\mathrm{Li}}}\right)^{5 / 6} .
\end{aligned}
$$

Let us note immediately that for laser parameters comparable with these required for thermonuclear fusion it is difficult to satisfy these inequalities for values $\kappa \sim \kappa_{\mathrm{st}}$ when

$$
\xi_{\mathrm{m}} \sim\left(L_{0} / \lambda_{0}\right)^{2 / 3}\left[\ln \left(\omega_{0} / v_{\mathrm{ei}}\right)\right]^{-1} .
$$

Since the size of the instability localization region is

$$
\Delta X \sim \xi_{\mathrm{m}}^{1 / 2}\left(\lambda_{0}^{2} L\right)^{1 / 3}
$$

the length of this parametric decay region is less than that for the other two instabilities considered by us above. This is connected with the strict resonance condition necessary for parametric decay. The absence of this resonance condition quickly reduces the parametric resonance situation to the case of aperiodic instability. The last remark indicate the exceedingly important role of the aperiodic parametric instability in the nonlinear wave transformation near the critical density where $\omega_{0} \simeq \omega_{\mathrm{Le}}$.

Now let us look at the results of the author and Starodub theory for one parametric instability of nonuniform plasma, which, if it can reduce to the absorption of the pump wave then apparently only during a limited period of time. Precisely here we should discuss the parametric capture of radiation by plasma.

We shall consider that parametric growing perturbations of the hf transverse electromagnetic field and aperiodic plasma field have a wavelength smaller that the wavelength of the pump field $\left(\kappa \gg \kappa_{0}\right)$. Then, the eiconal equation of such instability

$$
(t \rightarrow t+a)
$$

has the following form [12] :

$$
\begin{aligned}
\varepsilon^{\prime}(\omega, \kappa)=\frac{1}{4} & v_{\mathrm{E}}^{2}(X)\left(\kappa_{x}^{2}+\kappa_{z}^{2}\right)\left[\delta \varepsilon_{\mathrm{e}}^{1}(\omega, \kappa)+1\right] \delta \varepsilon_{\mathrm{i}}^{1}(\omega, \kappa) \times \\
& \times\left\{\left[\kappa^{2} c^{2}-\left(\omega-\omega_{0}\right)^{2} \varepsilon^{\mathrm{tr}}\left(\omega-\omega_{0}, \kappa\right)\right]^{-1}\right. \\
+ & {\left.\left[\kappa^{2} c^{2}-\left(\omega+\omega_{0}\right)^{2} \varepsilon^{\mathrm{tr}}\left(\omega+\omega_{0}, \kappa\right)\right]^{-1}\right\} }
\end{aligned}
$$

where

$$
\varepsilon^{\mathrm{tr}}(\omega, \kappa)=1+\sum_{\alpha} \delta \varepsilon_{\alpha}^{\mathrm{tr}}, \quad \varepsilon^{1}(\omega, \kappa)=1+\sum_{\alpha} \delta \varepsilon_{\alpha}^{\mathrm{l}}
$$

and $\delta \varepsilon_{\alpha}^{i r}, \delta \varepsilon_{\alpha}^{1}$-transverse and longitudinal partial dielectric permeabilities.

The space dependence of the pump field is primery. Its inherent length

$$
L_{\mathrm{E}}=\left(\hat{\lambda}_{0}^{2} L\right)^{1 / 3}
$$

is much less than $L$ (for the case $\lambda_{0} \ll L$ ). This permits us to use the approximation (1.17).

For the marginal conditions of instability the eiconal equation (1.25) has four solutions $\pm \kappa_{1}, \pm \kappa_{2}$ :

$$
\kappa_{x}=\kappa_{1,2}(X)=\kappa_{\perp} \sqrt{-p \pm \sqrt{p^{2}-q}} .
$$

Here,

$$
\begin{aligned}
& p(X)=1-\frac{v_{\mathrm{E}}^{2}(X) \omega_{\mathrm{Le}}^{2}(X)}{4 c^{2} \kappa_{\perp}^{2} \beta v_{\mathrm{Te}}^{2}}, \\
& q(X)=1+\frac{k^{4}}{\kappa_{\perp}^{4}}-\frac{v_{\mathrm{E}}^{2}(X) \kappa_{z}^{2}}{2 \kappa_{\perp}^{2} c^{2} r_{\mathrm{De}}^{2} \beta},
\end{aligned}
$$

where

$$
\begin{gathered}
\kappa_{\perp}^{2}=\kappa_{y}^{2}+\kappa_{z}^{2}, \quad k^{2}=2 \gamma_{t} \omega_{0} / c^{2}, \\
\beta=1+\left(r_{\mathrm{Di}} / r_{\mathrm{De}}\right)^{2}, \quad \gamma_{\mathrm{t}}=\frac{1}{2} v_{\mathrm{ei}} \omega_{\mathrm{Le}}^{2} / \omega_{0}^{2} .
\end{gathered}
$$

In the case where

$$
\kappa_{z}^{4}-\kappa_{y}^{4} \geqslant k^{4}=\left(2 \gamma_{t} \omega_{0} / c^{2}\right)^{2},
$$


there occurs parametric instability for the plasma perturbation localized by the turning points, and the marginal pump field is

$$
\begin{aligned}
& \frac{v_{\mathrm{E}}^{2}(m)}{v_{\mathrm{Te}}^{2}}=\frac{2 \beta c^{2}\left(\kappa_{\perp}^{4}+k^{4}\right)}{\kappa_{z}^{2} \omega_{\mathrm{Le}}^{2}(m)} \times \\
& \quad \times\left\{1+\frac{(2 n+1) \sqrt{\xi_{\mathrm{m}}}}{\left|\kappa_{z}\right| L_{\mathrm{E}}} \sqrt{\frac{2 \kappa_{\perp}^{2} \kappa_{z}^{2}}{\kappa_{\perp}^{4}+k^{4}}-1}\right\} .
\end{aligned}
$$

The length of the localization region in the vicinity of the $m$-th maximum of the pump field is

$\Delta \overline{\mathbf{X}}_{\mathrm{t}}(m)=\frac{2 L_{\mathrm{E}}}{\xi_{\mathrm{m}}^{1 / 4}} \sqrt{\frac{2 n+1}{\left|\kappa_{z}\right| L_{\mathrm{E}}}}\left(\frac{\kappa_{z}^{4}-\kappa_{y}^{4}-k^{4}}{\kappa_{\perp}^{4}+k^{4}}\right)^{1 / 4}$.

This region is small under condition $\kappa_{\perp} \gg \kappa_{0}$.

If $\kappa_{z}^{4}-\kappa_{y}^{4}<k^{4}$, then the localization of plasma perturbations is determined by its transformation in the branch points of the wave vector. In this case, we have the following expressions for the marginal field and for the length of localization :

$$
\begin{aligned}
\frac{v_{\mathrm{E}}^{2}(m)}{v_{\mathrm{Te}}^{2}}= & \frac{4 c^{2} \beta}{\omega_{\mathrm{Le}}^{2}(m)}\left[\kappa_{y}^{2}+\sqrt{\kappa_{y}^{4}+k^{4}}\right] \times \\
& \times\left\{1+\frac{(2 n+1) \sqrt{2 \xi_{\mathrm{m}}}}{L_{\mathrm{E}}\left(\kappa_{y}^{4}+k^{4}\right)^{1 / 4}} \sqrt{\frac{\sqrt{\kappa_{y}^{4}+k^{4}}-\kappa_{z}^{2}}{\sqrt{\kappa_{y}^{4}+k^{4}}+\kappa_{y}^{2}}}\right\}
\end{aligned}
$$

$$
\begin{aligned}
& \Delta \overline{\overline{\mathbf{X}}}(m)=\frac{2 L_{\mathrm{E}}}{\sqrt{\xi_{\mathrm{m}}}} \sqrt{\frac{(2 n+1) \sqrt{2 \xi_{\mathrm{m}}}}{L_{\mathrm{E}}\left(\kappa_{y}^{4}+k^{4}\right)^{1 / 4}}} \times \\
& \times\left(\frac{\sqrt{\kappa_{y}^{4}+k^{4}}-\kappa_{z}^{2}}{\sqrt{\kappa_{y}^{4}+k^{4}}+\kappa_{y}^{2}}\right)^{1 / 2} .
\end{aligned}
$$

Figure 1 schematically demonstrates the marginal pump field as a function of the wave vector $\kappa_{z}$ under assumption $\kappa_{y}=0$.

Let us note that the initial pump wave must not necessarily be a purely standing wave. For example the results for large $\kappa_{z}$ to be valid it is sufficient for that the reflection coefficient $R$ satisfy the condition :

$$
R>16(2 n+1)^{4} \xi_{\mathrm{m}}^{2}\left(\kappa_{\perp} L_{\mathrm{E}}\right)^{-4} .
$$

Since the trapped electromagnetic wave is a standing one it can be assumed that for a sufficiently large value of the oscillating electron velocity in the trapped field

$$
\tilde{v}_{\mathrm{E}} \sim 2 v_{\mathrm{Te}} \sqrt{\beta v_{\mathrm{ei}} / \omega_{\mathrm{o}}}
$$

there arises a new trapping instability, the result of which will be a new trapped electromagnetic field with shorter wavelength. Moreover, for the marginal field formulas

$$
\left(v_{\mathrm{E}} / v_{\mathrm{Te}}\right)^{2} \sim\left(\kappa_{\perp} \lambda_{0}\right)^{4}
$$

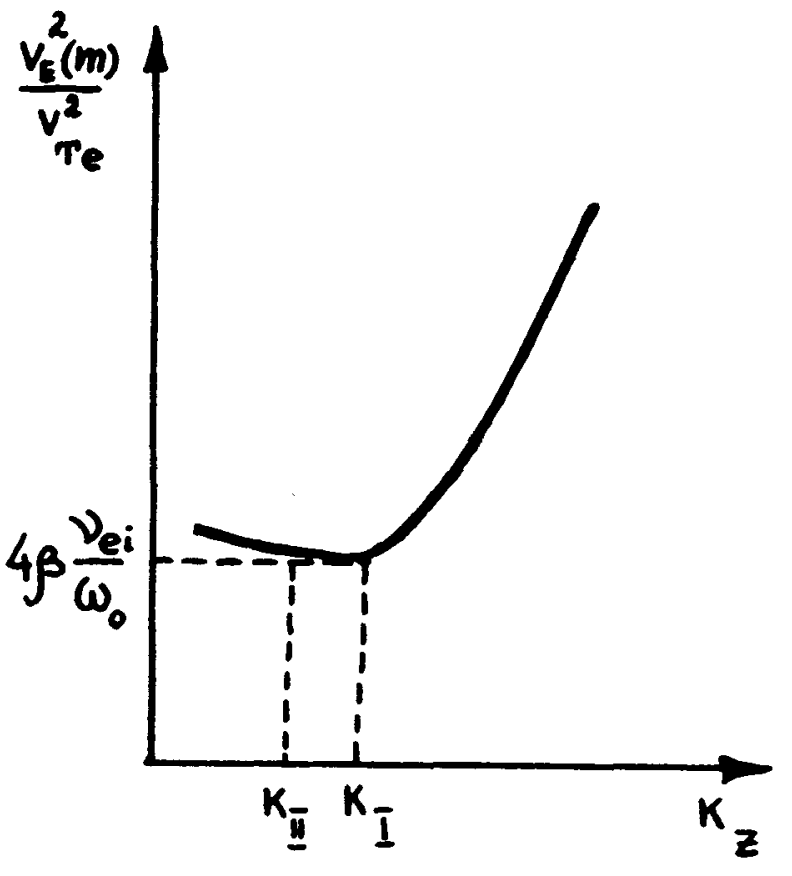

FIG. 1.

the scale of the pump field, much larger than the wavelength in vacuum, can be divided up to $t_{0}$. Therefore, one can assume that the most important result of this instability is the modification of the electromagnetic field structure $\left({ }^{2}\right)$.

In contrast to the trapping $t \rightarrow t+a$ instability the previously discussed parametric instabilities connected with the excitation of plamsons are characterised by a short wavelength plasma field. Thus it forms highly space changed electric fields much more singular than the pump wave field. The results of the statistical theory of such a plasma field are presented in the next part of our paper.

2. Parametric nonisothermal plasma turbulence and anamalous absorption of electromagnetic radiation by plasma. - Powerful electromagnetic radiation acting on plasma results in nonisothermality of plasma when ion acoustic waves exist. In particular, it is the reason why the process of Langmuir wave decay on Langmuir and ion acoustic waves becomes important in nonlinear dynamics resulting in a quasi-stationary turbulent state. This instability was examined in [14]. In [1] the role of this instability was underlined in nonlinear theory of the parametric resonance. Finally, under certain conditions, this process was studied in the parametric turbulence theory in papers $[15,16]$.

In our work [18] we take into account that due to the development of parametric instabilities arising under the action of an electromagnetic pumping wave there arises a Langmuir wave spectrum of finite

(2) Let us note that the assumptions of our paper [13] on the radiation capture due to stimulated Mandelstam-Brilluen scattering are not realized. 
bandwidth $\Delta \omega$. For example, when $\Delta \omega \gg \gamma_{\mathrm{s}} \gg \tilde{\gamma}$, it is the reason why the secondary instability $l \rightarrow l^{\prime}+s$ threshold is described by the relation

$$
\frac{E_{\mathrm{sc}, \mathrm{th}}^{2}}{8 \pi n_{\mathrm{e}} \kappa T_{\mathrm{e}}} \sim \frac{\tilde{\gamma} \Delta \omega}{\omega_{0} \omega_{\mathrm{s}}} .
$$

The spectral width $\Delta \omega$ is different for different initial parametric instabilities and increases with the threshold excess $p=E_{0} / E_{\text {th }}$.

The energy of an individual $n$-th satellite of Langmuir turbulence is described by the formula (compare [17]) :

$$
\frac{E_{n}^{2}}{8 \pi n_{\mathrm{e}} \kappa T_{\mathrm{e}}} \sim(\mathcal{N}+1-n) \frac{\tilde{\gamma} \Delta \omega}{\omega_{0} \omega_{\mathrm{s}}\left(\kappa_{\mathrm{d}}\right)}
$$

and the total number of satellites is

$$
\mathcal{N} \sim(\gamma / \tilde{\gamma})
$$

where $\kappa_{\mathrm{d}}$ is the wave number of the principal line arising due to primary parametric instability, $\gamma$ is the growth rate of this instability and is the function of the threshold excess. Therefore, the total energy of Langmuir turbulence is described by the formula

$$
\frac{E_{\text {long }}^{2}}{8 \pi n_{\mathrm{e}} \kappa T_{\mathrm{e}}} \sim \mathcal{N}^{2} \frac{E_{\mathrm{sc}, \text { th }}^{2}}{8 \pi n_{\mathrm{e}} \kappa T_{\mathrm{e}}} \sim \frac{\gamma^{2}\left(\kappa_{\mathrm{d}}\right) \Delta \omega}{\tilde{\gamma} \omega_{0} \omega_{\mathrm{s}}\left(\kappa_{\mathrm{d}}\right)} .
$$

According to this expression, the effective collision frequency can be determined by means of the usual formula

$$
v_{\mathrm{eff}} E_{0}^{2} \sim v_{\mathrm{ei}} E_{\text {long }}^{2} .
$$

This value characterizes the primary electromagnetic wave dissipation rate due to formation of a field of Langmuir waves and their damping determined by electron-ion collisions.

This theory is valid if the energy of the most intense line is less than the aperiodic instability threshold

$\frac{E_{1}^{2}}{8 \pi n_{\mathrm{e}} \kappa T_{\mathrm{e}}} \sim 8 \mathcal{N} \frac{\tilde{\gamma} \Delta \omega}{\omega_{0} \omega_{\mathrm{s}}}<\frac{\sqrt{\tilde{\gamma} \Delta \omega}}{\omega_{0}}$ i. e. $\frac{\gamma}{\omega_{\mathrm{s}}}<\sqrt{\frac{\tilde{\gamma}}{\Delta \omega}}$.

We shall discuss concrete parametric instabilities when this condition is satisfied and assumly resonance mismatch leading to the formation of plasmons with wave vectors satisfy the condition

$$
\kappa^{2} r_{\mathrm{De}}^{2} \sim \kappa_{\mathrm{st}}^{2} r_{\mathrm{De}}^{2} \sim\left[\ln \left(\omega_{0} / v_{\mathrm{ej}}\right)\right]^{-1}
$$

The two plasmon decay $(t \rightarrow 2 l)$ :

$$
\begin{aligned}
& \gamma \sim \Delta \omega \sim \omega_{0} \frac{v_{\mathrm{E}}}{c} \\
& v_{\mathrm{ef}}^{(21)} \sim \frac{\kappa T_{\mathrm{e}}}{m_{\mathrm{e}} c^{2}} \frac{\omega_{0}^{2} v_{\mathrm{E}}}{\omega_{\mathrm{s}} c} \lesssim \frac{\kappa T_{\mathrm{e}}}{m_{\mathrm{e}} c^{2}} \omega_{0}\left(\frac{v_{\mathrm{ei}}}{\omega_{\mathrm{Li}}}\right)^{1 / 3} .
\end{aligned}
$$

The aperiodic instability $(t \rightarrow l+a)$ :

$$
\begin{gathered}
\gamma \sim \Delta \omega \sim \omega_{0} \frac{v_{\mathrm{E}}^{2}}{v_{\mathrm{Te}}^{2}} \\
v_{\mathrm{ef}}^{(\mathrm{la})} \sim \frac{\omega_{0}^{2}}{\omega_{\mathrm{s}}}\left[\frac{E_{0}^{2}}{8 \pi n_{\mathrm{e}} \kappa T_{\mathrm{e}}}\right]^{2} \lesssim v_{\mathrm{ei}}\left(\frac{\omega_{\mathrm{Li}}}{v_{\mathrm{ei}}}\right)^{1 / 2} .
\end{gathered}
$$

The parametric decay $(t \rightarrow l+s)$ :

$$
\begin{gathered}
p>\sqrt{\gamma_{\mathrm{s}} / \tilde{\gamma}}>1 \\
\gamma \sim \Delta \omega \sim p \sqrt{\tilde{\gamma} \gamma_{\mathrm{s}}} ; \quad v_{\mathrm{ef}}^{(\mathrm{ls})} \sim 2 \gamma \lesssim v_{\mathrm{ei}}\left(\frac{\omega_{\mathrm{s}}}{v_{\mathrm{ei}}}\right)^{2 / 3} .
\end{gathered}
$$

The obtained results permit to determine the optical thickness conditioned by various mechanisms of the parametric instabilities. Thus for two-plasmon decay the optical thickness as a function of the radiation density of pump energy flux incident on the plasma we obtain

$$
\tau^{(21)} \sim 10^{-3} L \lambda_{0}^{-4 / 3} T_{\mathrm{e}}^{1 / 2} Z^{1 / 3}\left(q / q_{\max }^{(21)}\right)^{1 / 2} .
$$

Here, $Z=\left|e_{\mathbf{i}}\right| e \mid, \lambda_{0}=2 \pi \lambda_{0}$ is the pump wavelength, $L$ is the typical length of plasma inhomogeneity in $m \mu, T_{\mathrm{e}}$ is the electron temperature in $\mathrm{keV}$. The maximum value of floux density determined by the condition of theory validity is

$$
\frac{q_{\mathrm{max}}^{(21)}}{q_{\mathrm{th}}^{(21)}} \sim 20 T_{\mathrm{e}}^{2} \lambda_{0}^{4 / 3}, \quad q_{\mathrm{th}}^{(21)} \sim \frac{10^{11} Z^{2}}{T_{\mathrm{e}}^{3} \lambda_{0}^{4}} .
$$

Here the threshold energy flux is measured in $W / \mathrm{cm}^{2}$. The above formulas show that conditions with efficient absorption of neodym glass laser ligth can be realized due to two-plasmon decay in to day experiments. Under such conditions, $\mathrm{CO}_{2}$ laser radiation absorption can be less efficient.

The effective collision frequency determined by aperiodic instability can easily exceed the frequency of electron-ion collisions. In view of the relatively large size of turbulence region for this instability we can conclude that radiation anomalous absorption will determine light energy dissipation near the critical plasma density if the light energy flux exceeds by an order of magnetude the threshold value

$$
q_{\mathrm{th}}^{(\mathrm{la})} \sim \frac{10^{13} Z}{\lambda_{0}^{3} T_{\mathrm{e}}^{1 / 2}} .
$$

For the absolute parametric decay $t \rightarrow l+s$ instability, we have a less optimistic estimation of anomalous absorption. This is a consequence of plasma inhomogeneity which results in difficulties in realizing decay conditions for short waves with $\kappa \sim \kappa_{\text {st }}$ as a result of which the space region of this absolute instability decreases. On the other side there is an optimistic possibility to think about large space region of the convective parametric decay $t \rightarrow l+s$ 
instability and therefore about the high enough anomalous absorption rate.

Appendix. - The existence of localized waves is connected with the special position of the Stokes lines emerging from the turning points (1.18) and defined as

$$
\operatorname{Im} \int_{ \pm\left(\xi_{\mathrm{i}}-\xi_{\text {, }}\right)}^{\xi-\xi_{\mathrm{m}}} \kappa_{x} \mathrm{~d}\left(\xi-\xi_{\mathrm{m}}\right)=0 .
$$

In accordance with (1.20) and (1.21) the last equation has the following form

$$
\operatorname{Im} \int_{1}^{ \pm f} \mathrm{~d} \eta \sqrt{1-\eta^{2}}=0 .
$$

Here, the two signs correspond to two different Stokes lines, and

$$
f=C_{\mathrm{m}}^{1 / 2} \delta^{-1 / 2}\left(\xi-\xi_{\mathrm{m}}\right) \exp (i \pi / 8) .
$$

One Stokes line connects the turning points. It is the straight line inclined at an angle of $7 \pi / 8$ to the real axis of the plane of the complex variable $\xi-\xi_{m}$. Other Stokes lines are defined by equation

$$
\operatorname{Re} \int_{1}^{ \pm f} \sqrt{\eta^{2}-1} \mathrm{~d} \eta=0
$$

Near the turning points $(|\eta-1| \ll 1)$, we have

$$
\operatorname{Re}\left[\left(\xi-\xi_{\mathrm{m}}\right)^{3 / 2}( \pm \exp \{i \pi / 8\})^{3 / 2}\right]=0 .
$$

Since $\xi-\xi_{\mathrm{m}}=|r| e^{i \varphi}$, we can say that the Stokes lines are parallel to radii with

$$
\begin{aligned}
\varphi=-\frac{\pi}{8}-\frac{\pi}{2}[1-\operatorname{sign}( \pm 1)]+ & \\
& +\frac{\pi}{3}-\frac{2 \pi n}{3}, \quad n=0,1 .
\end{aligned}
$$

Therefore, the Stokes lines emerging from the right turning point are inclined to the real axis at angles of $5 \pi / 24$ and $-11 \pi / 24$ and consequently for the left turning point the Stokes lines are inclined at angles of $13 \pi / 24$ and $-19 \pi / 24$.

Such direction of the Stokes lines corresponds to the double connected external region of the turning points (1.18). Therefore, it is possible to use the BohrSommerfeld quantization rule for eiconal equation (1.16).

\section{References}

[1] Силин, В. П. Параметрическое воздействие излучения болышой моштности на плазму. Изд-во « Наука », Москва, 1973г.

SILIN, V. P., Parametricheskoe vosdeistvie izlucheniya bolshoi moshchnosti na plasmu (Parametric effect of High Power Radiation on Plasma) «Nauka» Ed., Moscow, 1973.

[2] Силин, В. П., Стародуб, А. Н., ЖЭТФ 66 (1974) 176. Srlin, V. P. and Starodub, A. N., Zh. Eksp. Teor. Fiz., 66 (1974), 176 [Sov. Phys. JETP (USA) 39 (1974) 82]

[3] Силин, В. П., Стародуб, А. Н., Филиппов, М. В., Препринт ФИАН n' 158,1976 г.

Silin, V. P., Starodub, A. N. and Filippov, M. V., (Physical Institute of the Academy of Sciences, Moscow, 1976, Preprint $\mathrm{n}^{\circ}$ 158).

[4] Силин, В. П., Стародуб, А. Н., Препринт ФИАН no 44, 1977 .

Silin, V. P. and STarodub, A. N. (Physical Institute of the Academy of Sciences, Moscow, 1977, Preprint $\left.n^{\circ} 44\right)$.

[5] JACKsON, E. A., Phys. Rev., 153 (1967) 235.

[6] LEe, Y. C. and KAw, P., Phys. Rev. Lett. 32 (1974) 135.

[7] Liu, C. S. and Rosenbluth, M. N., Phys. Fluids, 19 (1976), 967.

[8] Perkins, R. W. and Flick, J., Phys. Fluids, 14 (1971), 2012.

[9] Байков, И. С., Силин, В. П., ЖТФ 42 (1973) 3.

BaIkov, I. S. and Silin, V. P., Zh. Tekh. Fiz., 43 (1973), 3 [Sov. Phys.-Tech. Phys. (U. S. A.) 18 (1973), 1].
[10] Силин, В. П., Стародуб, А. Н., Физика ллазмві, 1977г, 3, 280.

Silin, V. P. and Starodur, A. N., Fizika Plazmy, 1977 (Soviet Journal of Plasma Phys., 1977).

[11] Силин, В. П., Стародуб, А. Н., Прелринт ФИАН, ${ }^{\circ} 110$, Москва, 19772.

Silin, V. P. and Starodub, A. N. (Physical Institute of the Academy of Sciences, Moscow, 1977, Preprint $n^{\circ} 110$ ).

[12] Горбунов, Л. М., Силин, В. П., ЖЭТФ 49 (1965) 1973. Gorbunov, L. M. and SiLIN, V. P., Zh. Eksp. Teor. Fiz 49 (1965), 1973 [Sov. Phys. JETP (USA) 22 (1966) 1347].

[13] Силин, В. П., Стародуб, А. Н., Письма в ЖЭТФ 23 (1976) 609.

Silin, V. P. and Starodub, A. N., Pisma Zh. Eksp. Teor. Fiz. 23 (1976), 609 [JETP Lett. (USA) 23 (1976), 559.

[14] Ораевский, В. Н., Сагдеев, Р. 3, ЖТФ 32 (1962) 1291.

OrAEVSKI, V. N. and SAGDEEV, R. Z., Zh. Tekh. Fiz. 32 (1962), 1291 [Sov. Phys.-Tech. Phys. (USA) 7 (1963), 955].

[15] Галеев, А. А., Ломинадзе, Д. Г., Мачабели, Г. В., ЖTФ 45 (1975) 1358.

Galeev, A. A., Lominadze, D. G., Machabeli, G. V., Zh. Tekh. Fiz. 45 (1975), 1358 [Sov. Phys.-Tech. Phys. (USA), 20 (1975) 862

[16] Yuen, S. Y., Phys. Fluids, 18 (1975), 1308.

[17] VAleo, E. and KRUER, W. L., Phys. Fluids, 16 (1973), 675.

[18] Быченков, В.Ю., Силин, В. П., Тихончук, В. Т., Письма в ЖЭТТФ, 26 (1977) 309.

Bychenkov, V. Yu., Silin, V. P. and TrhonchuK, V. T. Pisma Zh. Eksp. Teor. Fiz. (1977). 